\title{
Correlation of B-type Natriuretic Peptide with Severity of Coronary Artery Disease Assessed by SYNTAX Score II in ST Elevation Acute Coronary Syndrome Patients
}

\author{
Onur Tasar, Gonenc Kocabay', Metin Cagdas², Yavuz Karabag² \\ ${ }^{2}$ Department of Cardiology, Kars Kafkas University, Kars, Turkey \\ ORCID: \\ Onur Tasar: https://orcid.org/0000-0003-2030-3810 \\ Gonenc Kocabay: https://orcid.org/0000-0002-1439-0252 \\ Metin Cagdas: https://orcid.org/0000-0001-6704-9886 \\ Yavuz Karabag: https://orcid.org/0000-0002-8156-315X
}

Department of Cardiology, Elazig Research and Training Hospital, Elazig, 'Department of Cardiology, Kartal Kosuyolu Heart and Research Hospital, Istanbul,

\section{Abstract}

Background: We aimed to evaluate the role of B-type natriuretic peptide (BNP) in assessing severity of coronary artery disease by SYNTAX score (SS) II in a prospective study among ST elevation acute coronary syndrome (ACS) patients. Methods: One thousand and six patients with ST elevation myocardial infarction (STEMI) who admitted for primary percutaneous intervention were included. The patients were divided into two groups according to SS II values $(\leq 32$ and $>32)$. The independent predictors of high SS II were investigated, and the best cutoff value of BNP, high-sensitivity C-reactive protein (hs-CRP), peak troponin I, and hemoglobin level in predicting high SS II was determined. Results: There was a positive correlation between BNP, white blood cell, hs-CRP, fasting blood glucose, peak troponin I, and SS I. SS II and hemoglobin were negative, but other parameters were positively correlated. High SS II group independent predictors of hypertension, diabetes mellitus, smoking, multivessel disease, high Killip class, BNP, peak troponin I, hemoglobin, and hs-CRP levels were found in STEMI patients. The value of BNP $>87.15 \mathrm{pg} / \mathrm{ml}$ with $59 \%$ sensitivity and $77 \%$ specificity (area under the curve [AUC]: 0.722 [95\% confidence interval [CI]: $0.689-0.756], P<0.001$ ), hs-CRP $>10.85 \mathrm{mg} / \mathrm{dl}$ with $64 \%$ sensitivity and $64 \%$ specificity (AUC: 0.685 [95\% CI: $0.65-0.72$ ], $P<0.001$ ), peak troponin I $>77.83 \mathrm{ng} / \mathrm{mL}$ with $68 \%$ sensitivity and $63 \%$ specificity (AUC: 0.704 [95\% CI $0.67-0.738$ ], $P<0.001$ ), and hemoglobin $>16.75 \mathrm{~g} / \mathrm{dL}$ with $4 \%$ sensitivity and $97 \%$ specificity (AUC: 0.345 [95\% CI: $0.309-0.382] P<0.001$ ) independently predicted high SS II group. Conclusion: Serum BNP level was independently associated with the severity of coronary atherosclerosis in patients with ACS together with multivessel disease, left ventricular ejection fraction, hs-CRP, and troponin. Therefore, BNP assessment gives additional prognostic information for early risk stratification of patients with ACS.

Keywords: Acute coronary syndrome, B-type natriuretic peptide, coronary artery disease

\section{INTRODUCTION}

The SYNTAX score (SS) is an angiographic scoring system based on the severity and complexity of coronary lesions. ${ }^{[1,2]}$ To overcome the pitfalls of a system score based on coronary angiograms, seven clinical parameters, namely age, creatinine clearance $(\mathrm{CrCl})$, left ventricular ejection fraction (LVEF), presence of unprotected left main, peripheral

Received: 25-Jun-19 Revision: 18-Aug-19 Accepted: 01-Sep-19

Published Online: 25-Nov-19

\begin{tabular}{|l|l|}
\hline \multicolumn{3}{|c|}{ Access this article online } \\
\hline Quick Response Code: & Website: \\
& http://www.ijcva.com \\
\cline { 2 - 2 } & \\
\end{tabular}

vascular disease (PVD), female sex, and chronic obstructive pulmonary disease (COPD), have been added to SS to obtain SS II..$^{[3]}$

Address for correspondence: Dr. Onur Tasar Department of Cardiology, Elazig Research and Training Hospital, Elazig, Turkey. E-mail: tasaronur@hotmail.com

This is an open access journal, and articles are distributed under the terms of the Creative Commons Attribution-NonCommercial-ShareAlike 4.0 License, which allows others to remix, tweak, and build upon the work non-commercially, as long as appropriate credit is given and the new creations are licensed under the identical terms.

For reprints contact: reprints@medknow.com

How to cite this article: Tasar O, Kocabay G, Cagdas M, Karabag Y. Correlation of B-type natriuretic peptide with the severity of coronary artery disease assessed by SYNTAX score II in ST elevation acute coronary syndrome patients. Int J Cardiovasc Acad 2019;5:129-33. 
Cardiovascular biomarkers, such as B-type natriuretic peptide (BNP), high-sensitivity C-reactive protein (hs-CRP), and troponin $\mathrm{I}$, have been suggested to relate to coronary artery disease (CAD) severity. ${ }^{[4]}$ Furthermore, BNP has been used as a marker for prognosis in patients with acute coronary syndrome (ACS). ${ }^{[5-7]}$ Although several studies in ACS patients demonstrated an association between increased BNP levels and poor clinical outcome, ${ }^{[5,6]}$ it is not known if there is a relation between the quantity of BNP levels and the severity of CAD assessed by SS II.

We aimed to evaluate the role of BNP in assessing severity of CAD by SS II in a prospective study among ST elevation ACS patients.

\section{Methods}

This was a single-center study that was screened 1214 patients with ST elevation myocardial infarction (STEMI) who admitted for primary percutaneous intervention between January 2015 and March 2018. Exclusion criteria were previous myocardial infarction history, systemic inflammatory disease, hepatic disease, malignant disease, renal failure, and previous coronary artery bypass grafting. Finally, 1006 patients were included in the study. The research protocol was approved by our institution's ethics committee. All patients gave written inform consent. Clinical data, including sex, age, cardiovascular risk factors, medications, coronary angiogram, medical history, transthoracic echocardiography, and biochemical parameters, were collected. STEMI was defined as a presentation with acute chest pain, or suffocating shortness of breath, together with persistent ST-segment elevation $>1 \mathrm{~mm}$ in 2 or more adjacent leads, or with a new or presumed new left bundle branch block or right bundle branch block on electrocardiography. ${ }^{[8]}$ Hypertension (HT) was defined as repeated systemic blood pressure measurements exceeding 140/90 $\mathrm{mmHg}$ or treatment with antihypertensive drugs for a known diagnosis of HT. Diabetes mellitus (DM) was diagnosed by fasting blood glucose $\geq 126 \mathrm{mg} / \mathrm{dL}$, blood glucose $>200 \mathrm{mg} / \mathrm{dL}$ at any time, or a history of DM, including those treated with diet, oral medications, or insulin. Hypercholesterolemia was defined as a baseline cholesterol level $>200 \mathrm{mg} / \mathrm{dL}$ and/or a low-density lipoprotein cholesterol level $>130 \mathrm{mg} / \mathrm{dL}$ or previously diagnosed and treated hypercholesterolemia. Active smokers were those with regular smoking in the previous 6 months.

Baseline BNP, hs-CRP, and other routine blood tests were measured at admission. The BNP levels were measured by the enzyme immunoassay kit, based on standards and which ELISA technology (Biomedica Medizinprodukte $\mathrm{GmbH}$, Wien, Divischgasse) (normal $<100$ pg/ml). hs-CRP, lipid profile, and peak cardiac enzyme levels were also obtained from all patients. Emergent coronary angiography was performed by the Judkins technique (Siemens Axiom Artis zee 2010; Siemens Healthcare, Erlangen, Germany). Percutaneous coronary intervention (PCI) procedures were performed using standard techniques. The type of stents (bare metal or drug eluting) using the thrombectomy and balloon was also left to the operator's discretion. All patients were treated with aspirin, clopidogrel or ticagrelor, and unfractionated heparin with the recommended doses after hospital admission. During and after the procedures, the use of glycoprotein $2 \mathrm{~b} / 3 \mathrm{a}$ inhibitors was at the operator's choice. At the hospital, medical treatments of patients were performed according to international guidelines. ${ }^{[8]}$ At the admission, standard two-dimensional transthoracic echocardiography was performed for all patients (S5; GE Medical System, Horten, Norway). LVEF was measured by the Simpson method. The SS I and SS II were calculated according to baseline coronary angiography in all patients by two trained interventional cardiologists who were blinded to the patients' laboratory and clinical data. The SS I was determined for all coronary lesions with $>50 \%$ diameter stenosis in a vessel $>1.5 \mathrm{~mm}$, based on the SYNTAX and SS II calculator (www.syntaxscore.com). PVD was defined as aorta or arteries other than coronaries, with exercise-related claudication, or revascularization surgery, or reduced or absent pulsation measured by duplex ultrasonography, or angiographic stenosis of more than $50 \%$ in case of severe symptoms. COPD was defined as the long-term use of a chronic bronchodilator or steroids for lung disease. $\mathrm{CrCl}$ was defined by the Cockcroft-Gault formula, expressed in $\mathrm{ml} / \mathrm{min}$. The patients were divided into two groups according to SS II values ( $\leq 32$ and $>32$ ). All procedures performed in the study involving human participants were in accordance with the ethical standards of the institutional and/or national research committee and with the 1964 Helsinki declaration and its later amendments or comparable ethical standards. All patients provided written informed consent.

\section{Statistical analysis}

Data were analyzed using the SPSS 17.0 version (SPSS Inc., Chicago, Illinois, USA). Normality of the data was determined using the Kolmogorov-Smirnov test. The continuous variables with a normal distribution are presented as mean \pm standard deviation values, while those without a normal distribution are presented as median and interquartile range values. Frequency distribution was calculated for the categorical variables (numbers and percentages [\%]). The continuous variables of the two groups were compared using the Student's $t$-test or the Mann-Whitney U-test. Categorical data were compared using the Chi-square test or Fisher's exact test. Correlations between different variables were assessed by Pearson's correlation test for continuous variables and Spearman test for noncontinuous variables. Multivariate logistic regression analyses were performed to identify the independent predictors of high SS II, using variables that showed statistically significant association with them in the univariate analyses. Receiver operating characteristic curve analysis was used to determine the best cutoff value of BNP, hs-CRP, peak troponin I, and hemoglobin level in predicting high SS II. 


\section{RESULTS}

We included 1006 patients (mean age: $57.0 \pm 13.0$ years; $80 \%$ were male) who were diagnosed with STEMI and performed coronary angiography in our clinic. The ischemia-related artery was left anterior descending in 545 patients, right coronary artery in 336 patients, and circumflex artery in 112 patients. Multivessel disease was present in 432 patients. The SS II values of the patients ranged from 13.40 to 80.45 , and the median value was 30.5 . Patients were divided into two groups according to SS II values. The mean SS I and SS II values of the high SS II group were $18.0 \pm 5.0$ and $42.9 \pm 10.3$, respectively, whereas the mean SS and SS II values of the low SS II group were $15.4 \pm 3.7$ and $22.7 \pm 4.6$, respectively. The comparison of baseline clinical and laboratory parameters of patients is shown in Table 1. Patients in the high SS II group were older, more diabetic, more hypertensive, more PVD, more smokers, and a higher Killip class than the patients in the lower SS II group. In patients with high SS II group, the use of angiotensin-converting enzyme inhibitors, beta-blocker, and statin was higher, whereas the use of aspirin was similar in both the groups. Higher white blood cell (WBC), fasting blood glucose, peak troponin I, hs-CRP, BNP, lower hemoglobin, and glomerular filtration rate values were present in the high SS II group. Multivessel disease and left main coronary artery involvement were more frequent in the SS II high group, whereas the mean ejection fraction (EF) value in the SS II high group was lower.

When all patients were evaluated, there was a positive correlation between BNP, WBC count, hs-CRP, fasting blood glucose, peak troponin I, and SS I, whereas there was no correlation between hemoglobin and SS. SS II and hemoglobin were negative; on the other hand, other parameters were positively correlated. Patients were divided into the following three groups according to LVEF to reduce the effect of EF on BNP: EF low group with $<40 \%$, EF middle range group between $40 \%$ and $49 \%$, and high EF group with $\geq 50 \%$. In the restructured correlation analysis, it was observed that SS I and BNP were positively correlated outside the middle range group, and BNP and SS II were well correlated in all the EF groups. Relation between SS I and SS II with BNP, WBC count, hemoglobin, hs-CRP, fasting blood glucose, and peak troponin I is shown in Table 2.

In multivariate logistic regression analysis, high SS II group independent predictors of HT, DM, smoking, multivessel disease, high Killip class frequency, BNP, peak troponin I, hemoglobin, and hs-CRP levels were found in STEMI patients. Table 3 shows the independent predictors of the high SS II group.

The value of BNP $>87.15 \mathrm{pg} / \mathrm{ml}$ independently predicted high SS II group with $59 \%$ sensitivity and $77 \%$ specificity

\section{Table 1: Baseline characteristics}

\begin{tabular}{|c|c|c|c|c|}
\hline & All patients & SS II $\leq 32$ & SS II > 32 & $P$ \\
\hline Age of patient & $57.0 \pm 13.0$ & $50.8 \pm 9.9$ & $63.4 \pm 11.7$ & $<0.001$ \\
\hline Gender of patient (male) & $846.0(80.0)$ & $477.0(96.4)$ & $325.0(63.6)$ & $<0.001$ \\
\hline Presence of diabetes & $254.0(24.0)$ & $41.0(8.3)$ & $206.0(40.3)$ & $<0.001$ \\
\hline Presence of hypertension & $474.0(44.8)$ & $159.0(32.1)$ & $299.0(58.5)$ & $<0.001$ \\
\hline Presence of COPD & $47.0(4.4)$ & $12.0(2.4)$ & $35.0(6.8)$ & $<0.001$ \\
\hline Presence of peripheral arterial disease & $186.0(17.6)$ & $13.0(2.6)$ & $167.0(32.7)$ & $<0.001$ \\
\hline Presence of dyslipidemia & $425.0(40.2)$ & $216.0(43.6)$ & $196.0(38.4)$ & 0.089 \\
\hline Family history of CAD & $230.0(21.8)$ & $117.0(23.6)$ & $107.0(20.9)$ & 0.304 \\
\hline History of smoking & $556.0(52.6)$ & $330.0(66.7)$ & $203.0(39.7)$ & $<0.001$ \\
\hline Beta-blocker use & $86.0(8.1)$ & $30.0(6.1)$ & $55.0(10.8)$ & 0.007 \\
\hline Statin use & $197.0(18.6)$ & $81.0(16.4)$ & $111.0(21.7)$ & 0.031 \\
\hline Angiotensin-converting enzyme inhibitor/angiotensin receptor blocker use & $231.0(21.9)$ & $67.0(13.5)$ & $156.0(30.5)$ & $<0.001$ \\
\hline Previous medication aspirin use & $26.0(2.5)$ & $12.0(2.4)$ & $13.0(2.5)$ & 0.903 \\
\hline Killip class $>1$ on admission $(\%)$ & $218.0(20.6)$ & $53.0(10.7)$ & $158.0(30.9)$ & $<0.001$ \\
\hline WBC count $(/ 1000)$ & $12.4 \pm 4.0$ & $11.8 \pm 3.3$ & $13.0 \pm 4.6$ & $<0.001$ \\
\hline Hemoglobin (g/dL) & $13.6 \pm 1.9$ & $14.1 \pm 1.5$ & $13.1 \pm 2.1$ & $<0.001$ \\
\hline $\mathrm{CRP}(\mathrm{mg} / \mathrm{dl})$ & $10.8(5.9-18.3)$ & $7.7(4.8-13.5)$ & $14.0(7.7-24.5)$ & $<0.001$ \\
\hline Estimated glomerular filtration rate $\left(\mathrm{ml} / \mathrm{min} / 1.73 \mathrm{~m}^{2}\right)$ & $85.7 \pm 26.5$ & $98.3 \pm 20.6$ & $72.8(26.0)$ & $<0.001$ \\
\hline Fasting blood glucose on admission (mg/dL) & $128.0(105.0-172.0)$ & $116.0(100.0-134.0)$ & $148.0(118.0-216.0)$ & $<0.001$ \\
\hline Peak troponin I $(\mathrm{ng} / \mathrm{mL})$ & $84.4(38.8-187.0)$ & $54.4(21.7-117.7)$ & $121.0(56.0-237.3)$ & $<0.001$ \\
\hline $\mathrm{BNP}(\mathrm{pg} / \mathrm{mL})$ & $72.5(38.0-135.0)$ & $51.2(29.4-87.0)$ & $97.1(56.0-211.0)$ & $<0.001$ \\
\hline LVEF $(\%)$ & $46.4 \pm 8.7$ & $50.6 \pm 6.4$ & $42.4 \pm 8.7$ & $<0.001$ \\
\hline Left main CAD presence & $15.0(1.4)$ & $3.0(0.6)$ & $12.0(2.3)$ & 0.023 \\
\hline Multivessel disease presence & $431.0(40.8)$ & $153.0(30.9)$ & $256.0(50.1)$ & $<0.001$ \\
\hline Baseline SS & $16.7 \pm 4.6$ & $15.4 \pm 3.7$ & $18.0 \pm 5.0$ & $<0.001$ \\
\hline Baseline SS II & $33.0 \pm 12.9$ & $22.7 \pm 4.6$ & $42.9 \pm 10.3$ & $<0.001$ \\
\hline
\end{tabular}

COPD: Chronic obstructive pulmonary disease, SS: SYNTAX score, CAD: Coronary artery disease, WBC: White blood cell, CRP: C-reactive protein, LVEF: Left ventricular ejection fraction, BNP: B-type natriuretic peptide 


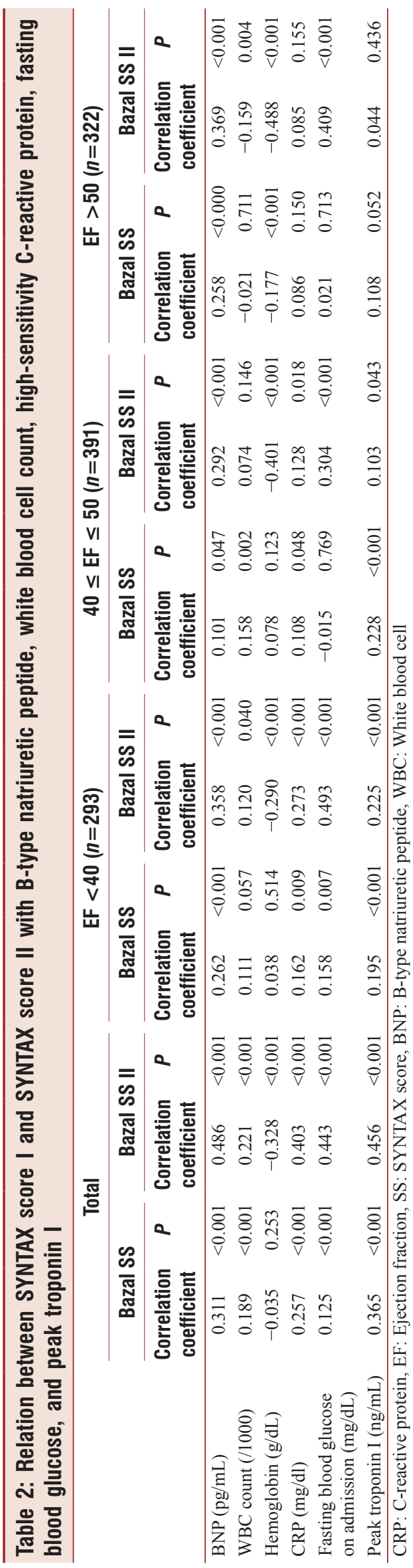

\begin{tabular}{|c|c|c|c|c|}
\hline & \multirow[t]{2}{*}{$P$} & \multirow[t]{2}{*}{ OR } & \multicolumn{2}{|c|}{$95 \%$ Cl for $\operatorname{Exp}(B)$} \\
\hline & & & Lower & Upper \\
\hline Presence of diabetes & $<0.001$ & 6.789 & 4.333 & 10.638 \\
\hline Presence of hypertension & $<0.001$ & 2.108 & 1.476 & 3.011 \\
\hline History of smoking & $<0.001$ & 0.534 & 0.372 & 0.766 \\
\hline Killip class $>1$ on admission ( $\%)$ & $<0.001$ & 2.260 & 1.393 & 3.667 \\
\hline Hemoglobin (g/dL) & $<0.001$ & 0.766 & 0.689 & 0.851 \\
\hline hs-CRP (mg/dl) & $<0.001$ & 1.036 & 1.018 & 1.054 \\
\hline Peak troponin I (ng/mL) & $<0.001$ & 1.005 & 1.003 & 1.007 \\
\hline Multivessel disease & $<0.001$ & 1.970 & 1.378 & 2.816 \\
\hline $\mathrm{BNP}(\mathrm{pg} / \mathrm{mL})$ & $<0.001$ & 1.004 & 1.002 & 1.006 \\
\hline
\end{tabular}

(AUC: 0.722 [95\% CI: 0.689-0.756], $P<0.001$ ); hs-CRP $>10.85 \mathrm{mg} / \mathrm{dl}$ independently predicted high SS II group with $64 \%$ sensitivity and $64 \%$ specificity (AUC: 0.685 [95\% CI: 0.65-0.72]) $P<0.001$ ); peak troponin I $>77.83 \mathrm{ng} / \mathrm{mL}$ independently predicted high SS II group with 68\% sensitivity and 63\% specificity (AUC: 0.704 [95\% CI: $0.67-0.738] P<0.001$ ); and hemoglobin $>16.75$ independently predicted high SS II group with $4 \%$ sensitivity and $97 \%$ specificity (AUC: 0.345 [95\% CI: 0.309-0.382] $P<0.001$ ).

\section{Discussion}

The main finding of this study is that BNP and hs-CRP levels are related to the severity of coronary atherosclerosis assessed by SS II: patients with multivessel disease showed higher BNP levels, and BNP threshold of $88 \mathrm{pg} / \mathrm{ml}$ is able to predict the extension and severity of coronary disease with $77 \%$ specificity. As far as we know, this article is the first in the literature that evaluates the relation between CAD severity by SS II and biomarker levels (BNP, hs-CRP, and troponin I).

SS I is an important tool that can help clinicians to establish the optimal revascularization approach in patients with CAD. SS II combines the anatomical-based SS with the clinical baseline variables. Seven clinical parameters (age, creatinine clearance, LVEF, presence of unprotected left main, PVD, female sex, and COPD) have been added to SS to obtain SS II. ${ }^{[3]} \mathrm{A}$ recent article published by Salvatore et al. ${ }^{[3]}$ reported that SS II might present a useful tool to predict the risk of adverse clinical events in patients with ACS and severe CAD undergoing PCI.

Previously, it has been showed that BNP provides predictive information on ACS, and it is related to the severity of CAD in patients with ACS. ${ }^{[9,10]}$ Palazzuoli et al. ${ }^{[5]}$ demonstrated that circulating BNP levels are elevated in ACS with diffuse coronary involvement and associated with multivessel disease and the extension of coronary disease. In addition, BNP could be indicators for multivessel disease, poor thrombolysis in myocardial infarction flow, and CAD extension. Although our results are in accordance with their study, we used SS II 
system as a different tool to assess the coronary artery severity. Besides the different scoring system, they found that BNP higher than $80 \mathrm{pg} / \mathrm{ml}$, which is lower than our findings, appears able to predict the extension of coronary disease. To calculate SS II, the anatomical SS is combined with age, $\mathrm{CrCl}$, LVEF, and COPD, all of which affect the BNP levels. According to the current study, this new value should be used with SS II scoring system.

Hs-CRP levels on admission are associated with short- and long-term mortality in patients with ACS. ${ }^{[11]}$ Karadeniz et al. ${ }^{[12]}$ found that serum hs-CRP levels could predict the severity and complexity of coronary atherosclerosis together with multivessel disease, LVEF, and troponin levels. Nevertheless, they used the SS I to assess the severity of atherosclerosis, and the cutoff point of 5.77 was found as an independent predictor of a high SS I in patients with ACS.

In addition, we investigated the relationship between SS II and clinical and laboratory variables by multiple regression analyses. We found that BNP, hs-CRP, peak troponin I levels as well as diabetes, smoking, and multivessel disease were independent predictors for the high SS II group.

\section{Limitations}

Long term follow up could not be possible because this is a cross sectional study. Although it is a single-center study, we recruited a large sample size.

\section{ConcLusion}

Serum BNP level was independently associated with the severity of coronary atherosclerosis in patients with ACS together with multivessel disease, LVEF, hs-CRP, and troponin. To our knowledge, this is the first study to demonstrate that BNP is an independent predictor of SS II in patients with ACS. Therefore, BNP assessment gives additional prognostic information for early risk stratification of patients with ACS.

\section{Financial support and sponsorship}

Nil.

\section{Conflicts of interest}

There are no conflicts of interest.

\section{RefEREnCES}

1. Akgun T, Oduncu V, Bitigen A, Karabay CY, Erkol A, Kocabay G, et al. Baseline SYNTAX score and long-term outcome in patients with ST-segment elevation myocardial infarction undergoing primary percutaneous coronary intervention. Clin Appl Thromb Hemost 2015;21:712-9.

2. Vroegindewey MM, Schuurman AS, Oemrawsingh RM, van Geuns RJ, Kardys I, Ligthart J, et al. Syntax score II predicts long-term mortality in patients with one- or two-vessel disease. PLoS One 2018;13:e0200076.

3. Salvatore A, Boukhris M, Giubilato S, Tomasello SD, Castaing M, Giunta R, et al. Usefulness of SYNTAX score II in complex percutaneous coronary interventions in the setting of acute coronary syndrome. J Saudi Heart Assoc 2016;28:63-72.

4. Gijsberts CM, Gohar A, Ellenbroek GH, Hoefer IE, de Kleijn DP, Asselbergs FW, et al. Severity of stable coronary artery disease and its biomarkers differ between men and women undergoing angiography. Atherosclerosis 2015;241:234-40.

5. Palazzuoli A, Maisel A, Caputo M, Fineschi M, Quatrini I, Calabrò A, et al. B-type natriuretic peptide levels predict extent and severity of coronary disease in non-ST elevation coronary syndromes and normal left ventricular systolic function. Regul Pept 2011;167:129-33.

6. Goyal BM, Sharma SM, Walia M. B-type natriuretic peptide levels predict extent and severity of coronary artery disease in non-ST elevation acute coronary syndrome and normal left ventricular function. Indian Heart J 2014;66:183-7.

7. Galvani M, Ottani F, Oltrona L, Ardissino D, Gensini GF, Maggioni AP, et al. N-terminal pro-brain natriuretic peptide on admission has prognostic value across the whole spectrum of acute coronary syndromes. Circulation 2004;110:128-34.

8. Ibanez B, James S, Agewall S, Antunes MJ, Bucciarelli-Ducci C, Bueno H, et al. 2017 ESC guidelines for the management of acute myocardial infarction in patients presenting with ST-segment elevation: The task force for the management of acute myocardial infarction in patients presenting with ST-segment elevation of the European Society of Cardiology (ESC). Eur Heart J 2018;39:119-77.

9. de Lemos JA, Morrow DA, Bentley JH, Omland T, Sabatine MS, McCabe $\mathrm{CH}$, et al. The prognostic value of B-type natriuretic peptide in patients with acute coronary syndromes. N Engl J Med 2001;345:1014-21.

10. Wei P, Wang HB, Fu Q, Bai J, Zhu Q. Levels of BNP and stress blood glucose in acute coronary syndrome patients and their relationships with the severity of coronary artery lesion. Cell Biochem Biophys 2014;68:535-9.

11. Mueller C, Buettner HJ, Hodgson JM, Marsch S, Perruchoud AP, Roskamm $\mathrm{H}$, et al. Inflammation and long-term mortality after non-ST elevation acute coronary syndrome treated with a very early invasive strategy in 1042 consecutive patients. Circulation 2002;105:1412-5.

12. Karadeniz M, Duran M, Akyel A, Yarlığlueş M, Öcek AH, Çelik İE, et al. High sensitive CRP level is associated with intermediate and high syntax score in patients with acute coronary syndrome. Int Heart J 2015;56:377-80. 